\title{
The system evaluation of DDP and fluorouracil in the nasopharyngeal carcinoma.
}

\author{
Lin Meng" ${ }^{\#}$ Mingming Yang*\#, Xingde Tian, Bi Qiang \\ Department of Otolarygngology Head and Neck Surgery, the First People's Hospital of Jingzhou, PR China \\ \#These authors contributed equally to this work
}

\begin{abstract}
Objective: To discuss the therapeutic effects of DDP and fluorouracil in Nasopharyngeal Carcinoma (NPC).

Methods: 40 patients with NPC who were treated in our hospital from January 2014 to August 2017 were chosen as the research objects and divided into two groups in line with the random number table. 40 patients in the control group were treated with the concurrent radiochemotherapy and 40 patients in the observation group were treated with DDP+fluorouracil (PF) combining with concurrent radiochemotherapy. Therapeutic effects, distant metastasis, local recurrence, five-year survival rate and untoward effects of patients in both groups were observed.

Results: 34 patents had Complete Remission (CR) and 5 patients had Partial Remission (PR) in the observation group. The total control ratio was $97.5 \%$. There were 26 patients with CR and 7 patients with PR in the control group. The total control ratio was $82.5 \%, \mathrm{P}<0.05$. Five-year OS and PFS in the observation group were $87.5 \%$ and $85 \%$, respectively and those of the control group were $75 \%$ and $\mathbf{8 2 . 5 \%}$, respectively, $P<0.05$. The occurrence of nausea and vomiting and leukocyte $\downarrow$ of patients in both groups had the significant difference, $P<0.05$. There was no significant difference in other untoward effects, $\mathbf{P}>\mathbf{0 . 0 5}$.

Conclusions: PF scheme concurrent radiochemotherapy significantly improved local control ratio of NPC and obviously enhanced five-year survival rate of patients, but untoward effects in the therapeutic process should be noticed to ensure curative effects.
\end{abstract}

Keywords: DDP, Fluorouracil, Nasopharyngeal carcinoma (NPC), Concurrent radiochemotherapy.

\section{Introduction}

NPC is a common malignocytoma of the head and neck. It is common in the south of China, South China and Southeast Asia. About 15-50 people in 100000 patients will attack every year [1]. NPC has the strong particularity in biological behavior. In the early stage of morbidity, clinical symptoms and signs are not obvious. As making a definite diagnosis, 70\% of patients have already belonged to the middle and late period. Radiotherapy is a first choice of NPC and maintain $50-60 \%$ of five-year overall survival for patients, but distant metastasis and local recurrence often result in therapeutic failure [2]. Inducing chemotherapy will reduce the tumor load in a short time, increase radiosensitivity, decrease the untoward effects, and form the obvious killing effects on the subclinical metastatic lesion in distant tissues and organs, so as to improve the local control ratio and disease free survival [3]. In order to discuss the therapeutic effects of DDP+fluorouracil (5-FU) (PF scheme) inducing chemotherapy for Intensity Modulated Radiation Therapy (IMRT), 40 patients with NPC were chosen to analyse their therapeutic process and results. The results were shown as follows.

\section{Data and Methods}

\section{General data}

40 patients with NPC who were treated from January 2014 to August 2017 in our hospital were chosen as research objects. Inclusion criteria: patients were diagnosed with NPC through the pathological examination, showing normal hematopoietic function, normal liver function, normal renal function, normal ECG, and measureable tumor lesions; pathological parting included non-cancroid or undifferentiated types; KPS $\geq 70$; clinical stages included stage III, stage IVa, and stage IVb. It is predicted that the lifetime exceeded 6 months; B ultrasound, Xray, CT and ECT examination verified that there was no distant metastasis. Patients were well-informed in this study and signed the informed consent form. Exclusion criteria: patients with contraindications in chemoradiotherapy; patients with NPC and other diseases; patients with history of chemotherapy 
before 1 month prior treatment; women in gestation period or lactating women; patients with history of serious cardiopulmonary disease. According to the random number table, these patients were divided into two groups. There were 29 male patients and 11 female patients in 40 patients of the observation group, which had the age of 48-76 y old and mean age of $(52.75 \pm 10.48 \mathrm{y}$ old $)$. There were 30 male patients and 10 female patients in 40 patients of the control group, which had the age of $45-75 \mathrm{y}$ old and $(51.68 \pm 9.27 \mathrm{y}$ old $)$. Both patients had no obvious difference in general data, $\mathrm{P}>0.05$.

\section{Therapeutic methods}

Patients in the control group were treated with the radiotherapy and patients in the observation group were treated with PF combining with concurrent radiochemotherapy.

Radiation methods: All patients were treated with IMRT. A thermoplastic film cover of the head, neck and shoulder was used to fix patients. CT scanning method was applied to scan from patients' head to the clavicle for $2 \mathrm{~cm}$. The depth of stratum was $3.3 \mathrm{~cm}$. The protective area was sketched. MRI enhancement scan and CT enhancement were used to scan and display the lump of patients in the nasopharynx. The prescription dosage in the target volume was set up as follows: GTVnx (nasopharynx lesion volume) 70-76 Gy, GTVnd (cervical lymph node lymphatic metastasis) 61-66 Gy, CTV1 (tumor target volume 1) 70 Gy, CTV2 54 Gy. After segmenting for 30 times, Varian 2300C linear accelerator $6 \mathrm{MV}$ X-ray was used to irradiate from the uniform or non-uniform angles, with the conventional segmentation, 2 Gy/times, 5 times/w.

Chemotherapy regimens: PF scheme was applied, DDP 80 $\mathrm{mg} / \mathrm{m}^{2}$, mixture $50 \mathrm{mml}$ normal saline, and $\mathrm{d} 1$ intravenous drip. Hydration was required before $1 \mathrm{~d}$ of pharmacy; 5 FU 500 $\mathrm{mg} / \mathrm{m}^{2}$, mixture $500 \mathrm{ml}$ normal saline, d1-5 intravenous drip; 1 times at the interval of $3 \mathrm{w}$. When patients had the serious untoward effects in the therapeutic process, dosage should be reduced. The reduced dosage was $20 \%$ of initial dosage. After adjustment, if there were serious untoward effects, patients should stop therapy. Patients in both groups should be treated for 2 periods.

\section{Observational indexes}

Therapeutic effects, distant metastasis, local recurrence, fiveyear survival rate and untoward effects of patients in both groups were observed.

The criteria of curative effects: According to WHO solid tumor's therapeutic evaluation, CR was judged: complete disappearance of tumor and no new lesion for more than $4 \mathrm{w}$; PR: by comparing with the pre-treatment, GTVnx was reduced by more than $50 \%$ and no new lesion for more than $4 \mathrm{w}$; SD (stability): by comparing with the pre-treatment, GTVnx was reduced by $50 \%$ or below or it was increased by no more than $25 \%$, and no new lesion for more than $4 \mathrm{w}$; PD (progression): by comparing with the pre-treatment, GTVnx was increased by more than $25 \%$ or had new lesions or had lesion metastasis. Tumor control ratio $=(\mathrm{CR}+\mathrm{PR}) /$ total patients $\times 100 \%$.

Toxic and side effects should be evaluated in line with NCI toxicant classification standard 3.0 version [4].

Five-year survival rate included overall survival and Progression-Free Survival (PFS).

\section{Statistical methods}

SPSS20.0 was applied to record, analyse and dispose. Measurement data were expressed as $(\overline{\mathrm{x}} \pm \mathrm{s})$, t-test; enumeration data were presented in $n, \chi^{2}$ test; $\mathrm{p}<0.05$ showed that there was the statistical difference.

\section{Results}

\section{Untoward effects of patients in both groups in treatment process}

The occurrence of nausea and vomiting and leukocyte $\downarrow$ of patients in both groups had the significant difference, $\mathrm{P}<0.05$. Other untoward effects had no significant difference, $\mathrm{P}>0.05$, shown in Table 1.

Table 1. The untoward effects of patients in both groups in treatment process.

\begin{tabular}{|c|c|c|c|c|c|c|c|c|c|c|c|}
\hline \multirow[t]{2}{*}{ Untoward effects } & \multicolumn{5}{|c|}{ Observation group $(n=40)$} & \multicolumn{5}{|c|}{ Control group $(n=40)$} & \multirow[t]{2}{*}{$\mathbf{P}$} \\
\hline & $\mathbf{I}$ & II & III & IV & OS (\%) & I & II & III & IV & OS (\%) & \\
\hline Nausea and vomiting & 19 & 12 & 5 & 0 & 90.0 & 17 & 4 & 0 & 0 & 52.5 & 0.000 \\
\hline Blood platelet $\downarrow$ & 12 & 9 & 1 & 3 & 62.5 & 11 & 7 & 6 & 0 & 60.0 & 0.713 \\
\hline Leukocyte $\downarrow$ & 5 & 11 & 20 & 1 & 92.5 & 8 & 14 & 9 & 0 & 77.5 & 0.003 \\
\hline Hemoglobin $\downarrow$ & 6 & 13 & 7 & 0 & 65.0 & 5 & 14 & 5 & 0 & 60.0 & 0.465 \\
\hline Glutamic-pyruvic transaminase & 7 & 9 & 1 & 0 & 42.5 & 6 & 8 & 1 & 0 & 37.5 & 0.470 \\
\hline Urea nitrogen & 5 & 3 & 2 & 0 & 25.0 & 6 & 2 & 1 & 0 & 22.5 & 0.677 \\
\hline Oral mucositis & 10 & 11 & 13 & 1 & 87.5 & 23 & 8 & 5 & 1 & 92.5 & 0.239 \\
\hline Radiodermatitis & 19 & 12 & 5 & 2 & 95.0 & 14 & 26 & 4 & 3 & 97.5 & 0.352 \\
\hline
\end{tabular}




\section{Clinical effects of patients in both groups}

There were 34 patients with CR and 5 patients with PR in the observation group. The total control ratio was $97.5 \%$. There were 26 patients with $\mathrm{CR}$ and 7 patients with PR in the control group. The total control ratio was $82.50 \%, \mathrm{P}<0.05$, Table 2 .

Table 2. Clinical effects of patients in both groups.

\begin{tabular}{lllllll}
\hline Groups & Cases & CR & PR & SD & PD & Total control ratio (\%) \\
\hline $\begin{array}{l}\text { Observatio } \\
\text { n group }\end{array}$ & 40 & 34 & 5 & 1 & 0 & 97.5 \\
\hline $\begin{array}{l}\text { Control } \\
\text { group }\end{array}$ & 40 & 26 & 7 & 5 & 2 & 82.5 \\
\hline$X^{2}$ & $/$ & 4.267 & 0.392 & 2.883 & 2.051 & 12.500 \\
\hline$P$ & $/$ & 0.039 & 0.531 & 0.090 & 0.152 & 0.000 \\
\hline
\end{tabular}

\section{One-year and five-year survival rates of patients in both groups}

One-year OS and PFS in the observation group were $92.5 \%$ and $90 \%$, respectively and those of the control group were $90 \%$ and $85 \%$, respectively. Five-year OS and PFS in the observation group were $87.5 \%$ and $85 \%$, respectively and those of the control group were $75 \%$ and $82.5 \%$, respectively, $\mathrm{P}<0.05$, Table 3 .

Table 3. One-year and five-year survival rates in both groups.

\begin{tabular}{|c|c|c|c|c|c|}
\hline \multirow[t]{2}{*}{ Groups } & \multirow[t]{2}{*}{ Cases } & \multicolumn{2}{|c|}{ One-year survival rate (\%) } & \multirow{2}{*}{$\begin{array}{l}\text { Five-year } \\
(\%)\end{array}$} & \multirow{2}{*}{$\begin{array}{l}\text { survival rate } \\
\text { PFS }\end{array}$} \\
\hline & & os & PFS & & \\
\hline $\begin{array}{l}\text { Observatio } \\
\text { n group }\end{array}$ & 40 & 92.5 & 90.0 & 87.5 & 85.0 \\
\hline $\begin{array}{l}\text { Control } \\
\text { group }\end{array}$ & 40 & 90.0 & 85.0 & 75.0 & 72.5 \\
\hline$x^{2}$ & l & 0.391 & 1.143 & 5.128 & 4.669 \\
\hline$P$ & I & 0.532 & 0.285 & 0.024 & 0.031 \\
\hline
\end{tabular}

\section{Discussions}

NPC has the relatively higher sensitivity to radiotherapy and chemotherapy. The five-year survival rate of pure radiotherapy was only $67-77 \%$ [5], so inducing chemotherapy combining with the concurrent radiochemotherapy is standard and widely applied treatment mode.

This study indicated that there were 34 patients with CR and 5 patients with PR in the observation group. The total control ratio was $97.50 \%$. There were 26 patients with $\mathrm{CR}$ and 7 patients with $\mathrm{PR}$ in the control group. The total control ratio was $82.50 \%, \mathrm{P}<0.05$. Five-year OS and PFS in the observation group were $87.5 \%$ and $85 \%$, respectively and those of the control group were $75 \%$ and $82.5 \%$, respectively, $\mathrm{P}<0.05$. The occurrence of nausea and vomiting and leukocyte $\downarrow$ of patients in both groups had the significant difference, $\mathrm{P}<0.05$. Other untoward effects had no significant difference, $\mathrm{P}>0.05$.

Concurrent radiochemotherapy can reduce death possibility of NPC patients by $40-53 \%$ [6], because the local control ratio can be effectively enhanced. Meanwhile, it can reduce distant metastasis and increase radiosensitivity. PF scheme is the most extensive scheme in clinical NPC inducing chemotherapy and it is a recommended drug in concurrent radiochemotherapy of NPC. Inducing chemotherapy neoadjuvant chemotherapy is the chemotherapy before radical radiation therapy [7]. Through the concurrent radiochemotherapy, the cytotoxic effect in new drugs can reduce tumor, improve anoxia of engine body, promote synchronization of cell cycles, enhance radiosensitivity, show the interference or inhibiting effect on the sublethal cell injury of tumor cells, so as to develop the synergistic effects on the radiotherapy or even wipe out the subclinical metastatic lesion. Moreover, chemical toxicity in treatment won't generate the superposition effects with radiation toxicity, reduce the radiotherapy toxicity, and effectively improve local control ratio of NPC. Inducing chemotherapy has no obvious research results on improving the patients' survival rate. Some researchers think that inducing chemotherapy can significantly enhance patients' five-year disease free survival [8]. Some researchers also think that it can significantly improve patients' total survival rate and disease free survival $[9,10]$. Concurrent radiochemotherapy will increase toxic and side effects, while enhancing patients' local control ratio and survival rate. Reduction of leucopenia and nausea and vomiting are two common untoward effects. In the treatment process, it is necessary to reinforce symptomatic treatment to ensure the therapeutic effects. With the progress of the target treatment, some scholars combine concurrent radiochemotherapy with the targeted drug, thus the treatment will be more accurate. This reduces the toxic and side effects, while improving the curative effects.

To some up, PF scheme concurrent radiochemotherapy significantly improved local control ratio of NPC and obviously enhanced five-year survival rate of patients, but untoward effects in the therapeutic process should be noticed to ensure curative effects.

\section{References}

1. Joseph TSW, Tam CH, Susan LL. Is nasopharyngeal cancer really a Cantonese cancer? Chin J Cancer 2010; 29: 517-526.

2. Lee AWM, Sze WM, Au JSK. Treatment results for nasopharyngeal carcinoma in the modern era: The Hong Kong experience. Int J Rad Oncol Biol Phys 2005; 61: 1107-1116. 
3. Du Q, Wang R. The research progress of the inducing chemotherapy for NPC. Guangdong Med 2012; 33: 2685-2688.

4. Trotti A, Colevas AD, Setser A, Rusch V, Jaques D, Budach V, Langer C, Murphy B, Cumberlin R, Coleman CN, Rubin P. CTCAE v3.0: development of a comprehensive grading system for the adverse effects of cancer treatment. Sem Rad Oncol 2003; 13: 176-181.

5. Yi JL. Nasopharyngeal carcinoma treated by radical radiotherapy alone: Ten-year experience of a single institution. Int J Rad Oncol Biol Phys 2006; 65: 161-168.

6. Lee AW, Tung SY, Chan AT. Preliminary results of a randomized study (NPC-9902 Trial) on therapeutic gain by concurrent chemotherapy and/or accelerated fractionation for locally advanced nasopharyngeal carcinoma. Int $\mathrm{J}$ Radiat Oncol Biol Phys 2006; 66: 142-151.

7. Cirillo M, Venturini M, Ciccarelli L. Clinician versus nurse symptom reporting using the National Cancer InstituteCommon Terminology Criteria for Adverse Events during chemotherapy: results of a comparison based on patients self-reported questionnaire. Ann J Eur Soc Med Oncol 2009; 20: 1929-1935.
8. Jun MA, Mai HQ, Hong MH. Long-term results of a prospective randomized trial comparing neoadjuvant chemotherapy plus radiotherapy with radiotherapy alone for patients with locoregionally advanced nasopharyngeal carcinoma. Chinese J Cancer 2001; 19: 1350-1357.

9. Forastiere AA. Head and neck cancer: overview of recent developments and future directions. Semin Oncol 2000; 27: $1-4$.

10. Tan T, Lim WT, Fong KW. Concurrent chemo-radiation with or without induction gemcitabine, carboplatin, and paclitaxel: a randomized, phase $2 / 3$ trial in locally advanced nasopharyngeal carcinoma. Int $\mathrm{J}$ Rad Oncol Biol Phys 2015; 91: 952-960.

\section{*Correspondence to}

Mingming Yang

Department of Otolarygngology Head and Neck Surgery

The First People's Hospital of Jingzhou

PR China 